\title{
Degeneration of three or more lumbar discs significantly decreases lumbar spine/ hip ROM ratio during position change from standing to sitting in AVN patients before THA
}

\author{
Jianming Gu, Huixiong Feng, Xiao Feng and Yixin Zhou* id
}

\begin{abstract}
Background: Limitations in the lumbar spine movement reduce lumbar vertebral motion and affect spinopelvic kinematics. We studied the influence of lumbar intervertebral disc degeneration on spinofemoral movement, from standing to sitting, in patients undergoing total hip arthroplasty (THA).

Methods: Of 138 consecutive patients scheduled for THA due to unilateral avascular necrosis (AVN) of the femoral head, those with $\geq 3$ discs with University of California at Los Angeles (UCLA) disc degeneration score $>1$ were defined as the lumbar degenerative disc disease (LDD) group, and the remaining patients constituted d the control group. Full body anteroposterior and lateral EOS images in the standing and sitting positions were obtained. Pelvic incidence (PI), L1 slope (L1 s), lumbar lordosis angle (LL), pelvic tilt (PT), sacral slope (SS), femoral slope (Fs), sagittal vertical axis (SVA), hip flexion, lumbar spine flexion, and total spinofemoral flexion were measured on the images and compared between groups.

Results: No significant between-group differences were observed in the height, weight, body mass index, AVN staging, or PI, SS, and Fs on standing. The LDD group included more females and older patients, had $5^{\circ}$ lesser LL, $5^{\circ}$ greater PT, and larger SVA. From standing to sitting, the PI remained constant in both groups. Total spinofemoral flexion was $7^{\circ}$ less, lumbar spine flexion $16^{\circ}$ less, L1 slope change $6^{\circ}$ less, and SS change $8^{\circ}$ less, and hip flexion was $7^{\circ}$ more in the LDD than in the control group. The spine/hip flexion ratio was significantly lower in the LDD group $(0.3$ versus $0.7 ; p<0.001)$. On regression analysis, the LDD group $(p<0.001)$ and older age $(p=0.048)$ but not sex, weight, or height were significant univariate predictors of decreased spine/hip ratio.

Conclusions: Patients with LDD leant more forward and had a larger pelvis posterior tilt angle on standing and a decreased lumbar spine/hip flexion ratio, with more hip joint flexion, on sitting, to compensate for reduced lumbar spine flexion. Surgeons should be aware that elderly patients with multiple LDD have significantly different spinofemoral movements and increased risk of posterior dislocation post-THA. Preoperative patient identification, intraoperative surgical technique modification, and individualized rehabilitation protocols are necessary.
\end{abstract}

Keywords: Avascular necrosis; hip-spine syndrome, Sagittal balance, Total hip arthroplasty

* Correspondence: orthoyixin@yahoo.com

Department of Orthopedics, Beijing Jishuitan Hospital, Fourth Clinical College of Peking University, Beijing 100035, China

(c) The Author(s). 2020 Open Access This article is distributed under the terms of the Creative Commons Attribution 4.0 International License (http://creativecommons.org/licenses/by/4.0/), which permits unrestricted use, distribution, and reproduction in any medium, provided you give appropriate credit to the original author(s) and the source, provide a link to the Creative Commons license, and indicate if changes were made. The Creative Commons Public Domain Dedication waiver (http://creativecommons.org/publicdomain/zero/1.0/) applies to the data made available in this article, unless otherwise stated. 


\section{Background}

Accurate orientation of hip components is essential during total hip arthroplasty (THA) to avoid impingement and to maintain stability during daily activities. Different definitions are available in literature for safe zones for acetabular components. Kummer et al. (1999) reported that adequate ranges of cup inclination and anteversion were $35-45^{\circ}$ and $0-10^{\circ}$, respectively [1]. The classic safe zones $\left(40^{\circ} \pm 10^{\circ}\right.$ inclination and $15^{\circ} \pm 10^{\circ}$ anteversion in reference to the anterior pelvic plane) advocated by Lewinneck in 1978 have been widely applied by surgeons [2]. Widmer et al. (2004) reported that the sum of cup anteversion and 0.7 times the stem anteversion should be $37.3^{\circ}$ to achieve maximal and stable postoperative hip range of motion (ROM) [3]. However, Abdel et al. (2016) reported that in more than $60 \%$ of 206 cases of dislocation after THA, acetabular components in the "safe zone" were noted [4].

The functional angles of the acetabular component in THA patients may change according to the body posture [5-8]. Studies have reported a $22.3-28.7^{\circ}$ posterior pelvic tilt change from standing to sitting [6-8]. Babisch et al. (2008) reported that for every $1^{\circ}$ change in the pelvic tilt, the cup inclination and anteversion changed by approximately $0.3^{\circ}$ and $0.8^{\circ}$, respectively [5]. This pelvic movement functions as a protective mechanism against posterior dislocation when sitting or squatting. However, the limitation in lumbar spine motion may reduce this dynamic protection.

In addition to lumbar spine fusion, multiple lumbar disc degeneration (LDD) decreases the lumbar spine movement. McGregor et al. (1997) reported that patients with a degenerative lumbar spine or disc prolapse have an approximately $10^{\circ}$ decrease in flexion and extension compared to the age-matched controls [9]. Lumbar spine movement reduction results in greater risk of dislocation and revision after THA $[10,11]$. Elucidation and measurement of the effects of lumbar disc degeneration on spinopelvic movement are, thus, essential when preparing patients for THA. Therefore, this study investigated the effects of multiple lumbar intervertebral disc degeneration on spinofemoral movement during position change from standing to sitting in patients scheduled for hip joint replacement.

\section{Methods}

\section{Study participants}

With the approval of our institutional review board, 138 consecutive patients with unilateral avascular necrosis of femoral head (AVNFH) scheduled for primary THA from June to November 2018 were included in this retrospective study. AVNFH was staged according to the criteria of Ficat [12]. Verbal informed consent was obtained from the participants or their guardians.
The exclusion criteria were as follows: (1) hip diseases other than AVNFH, such as ankylosing spondylitis, developmental dysplasia of the hip, or rheumatoid arthritis, (2) squatting limitation (torso and hip flexion angle < $90^{\circ}$ ), (3) previous hip surgery, including hip arthroplasty, osteotomy, or osteosynthesis, (4) presence of lumbar scoliosis with an L1-S1 Cobb angle $>10^{\circ}$, and (5) history of spine compression fracture, spondylolysis, or spinal fusion surgery.

\section{Image acquisition}

All patients underwent biplanar full body examination using low radiation dose EOS imaging (EOS Imaging, Paris, France) in preparation for hip surgery [13]. Anteroposterior (AP) and lateral images in both standing and sitting positions (Fig. 1) were obtained and reconstructed using EOS Stereos ${ }^{\circ}$ software [14]. Standing and sitting positions were standardized for all subjects. Patients were imaged with their femora aligned approximately parallel to the floor, seated at $90^{\circ}$ of apparent hip flexion. The degrees of true flexion of the spine and hip were calculated using the imaging system. This relaxed seated position has been used in previous studies $[15,16]$.

Spine and pelvic sagittal parameters were measured using the EOS Stereos software and Surgimap software [17]. All measurements were performed by two trained researchers. In cases of greater than $10 \%$ mismatch between observers, another review was performed by a separate observer, and measurements were repeated and discussed till a consensus was reached.

\section{Parameter measurements \\ Group allocation}

Lumbar degeneration severity was evaluated using the UCLA disc degeneration scale $[18,19]$. Disk space narrowing, osteophyte formation, and endplate sclerosis were graded as 2, 3, and 4 points, respectively. Disks without these aforementioned changes were graded as 1 point. We defined the lumbar degenerative disc disease (LDD) group as patients with three or more discs graded $>1$ point. Patients with fewer than three discs graded $>1$ point were allocated to the control group.

\section{Anatomic parameters}

Pelvic incidence (PI) was defined as the angle between the perpendicular plane to the middle of the upper plate of $\mathrm{S} 1$ and the line joining this point to the bicoxofemoral axis.

\section{Positional parameters}

L1 slope (L1 s) was defined as the angle between the line along the L1 superior endplate and the reference horizontal line. The lumbar lordosis (LL) was measured from the superior endplate of L1 to the superior endplate of 


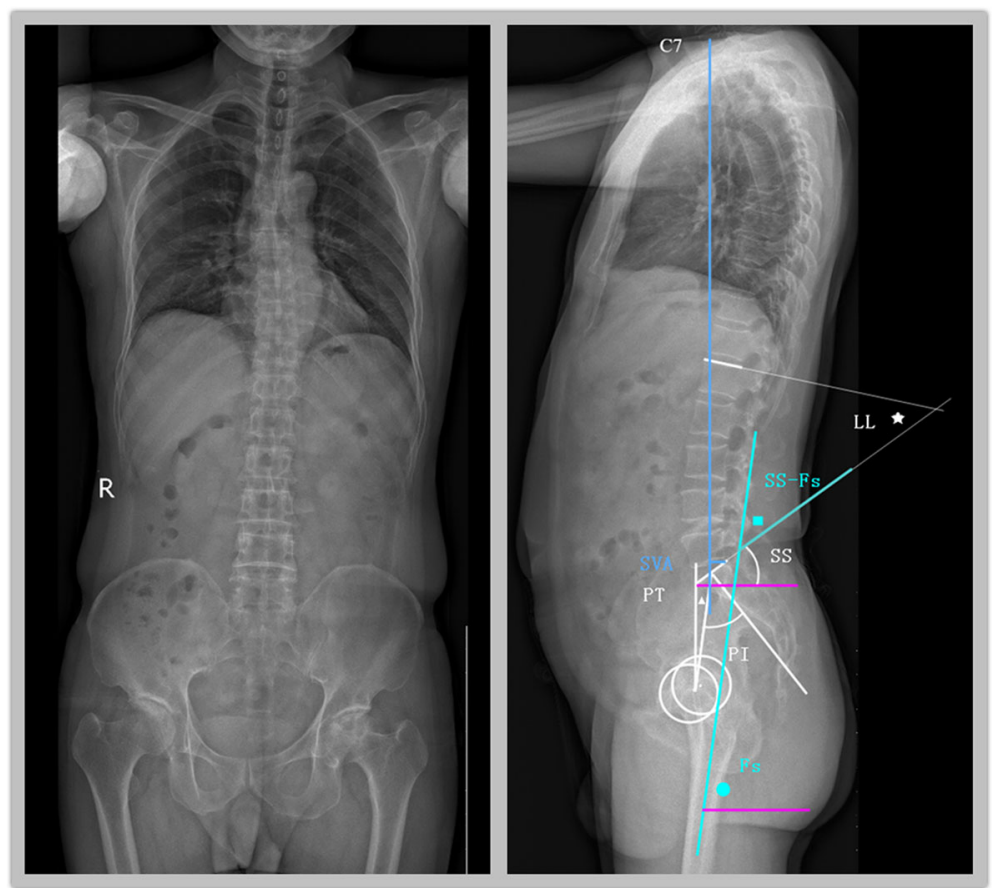

Fig. 1 Anteroposterior and lateral EOS imaging and spinopelvic parameters. 1) Pelvic incidence (PI): the angle between the perpendicular plane to the middle of the S1 upper endplate and the line joining this point to the bicoxofemoral axis. 2) Sacral slope (SS): the angle between the horizontal plane and the upper endplate of S1. 3) Pelvic tilt (PT, $\mathbf{\Delta}$ ): the angle of the line connecting the middle S1 upper plate and bicoxofemoral axis to the vertical axis. 4) Lumbar lordosis angle ( $L L, \star \star)$ : the angle between the superior endplates of $L 1$ and $S 1$. 5) Femoral slope $(F s, 0)$ : the angle between the axis of the femur and horizontal plane. Hip flexion is expressed as the change in the angle (SS-Fs, $\square$ ) between SS and Fs. 6) Sagittal vertical axis (SVA): the horizontal distance from the C7 plumb line to posterior superior corner of the superior margin of S1

S1. Lordosis was expressed as a positive value and kyphosis as a negative value. The changes in LL during a position change from standing to sitting were calculated as lumbar spine ROM (lumbar spine flexion). (3) The sacral slope (SS) was defined as the angle between the horizontal plane and superior endplate of S1. The SS is a positional parameter that varies according to pelvis positioning: changes in SS indicate pelvic rotation. Posterior rotation (anterior superior iliac spine moving backwards) was recorded as a positive value, and anterior rotation was recorded as a negative value. Pelvic tilt (PT) was defined as the angle of the line connecting the middle S1 upper plate and the bicoxofemoral axis to the vertical axis. Femoral slope (Fs) was defined as the angle between the axis of the proximal femur and the horizontal plane, with changes in Fs indicating changes in femur position. Hip flexion was defined as movement between the femur and acetabulum of the pelvis, and was evaluated as the change in the angle between the superior S1 endplate and the proximal femur axis (SS-Fs). The total flexion of the hip-spine complex was defined as the change in the angle between the L1 superior endplate and the proximal femur axis, and comprised lumbar spine flexion (change in LL) and hip flexion (change in SS-Fs). The spine/hip flexion ratio was calculated as lumbar spine flexion divided by hip flexion. The sagittal vertical axis (SVA) was defined as the horizontal distance from the $\mathrm{C} 7$ plumb line to the posterior superior corner of the superior margin of S1 (Fig. 1).

\section{Data analysis}

The normality of data distribution was determined using the Kolmogorov-Smirnov test. Radiographic parameters were compared using the Student's $t$-test for continuous data and Fisher's exact test for binominal data. Regression analyses were conducted to identify relationships between the spine/hip ratio and other variables. Data have been expressed as mean \pm standard deviation. All data were analyzed with SPSS (version 21.0; IBM SPSS, Chicago, IL, USA) software. A $P$-value of $<0.05$ was considered statistically significant.

\section{Results}

A total of 138 cases of AVN were enrolled in this study (control group, $n=84$; LDD group, $n=54$ ). Overall, the mean age of the patients was $55.4 \pm 12.9$ years, and the mean body mass index (BMI) was $26.0 \pm 9.2 \mathrm{~kg} / \mathrm{m}^{2}$. There were more female (52.9\%) than male patients, and the LDD group included significantly more females than the control group $(p=0.025)$. Most patients had Ficat 
Stage III AVN, and the AVN stage was comparable between the groups $(p=0.557)$. There were no significant differences in height, weight, or BMI between the groups. The demographic characteristics of the groups are shown in Table 1.

On standing, no significant differences were identified in PI, SS, or Fs between THE two groups. The LDD group had $5^{\circ}$ lesser LL and a $2^{\circ}$ smaller L1 slope than the control group. The PT was $5^{\circ}$ greater in the LDD than in the control group. The SVA in the LDD group was $29 \mathrm{~mm}$ greater, indicating that the center of gravity was more anterior to the femoral head and the upper bodies of these patients leant more forward than those in the control group.

On changing position from standing to sitting, the femur rotated from a vertical to a horizontal position. The femur rotated $86.2^{\circ}$ on an average; no significant difference was observed between the groups. The mean change in PI was minimal $\left(1^{\circ}\right)$, indicating a constant anatomical parameter that was not influenced by position. At the distal end of the lumbar spine, no significant difference was observed in the SS between the groups during standing. In contrast, at the proximal end of the lumbar spine, the L1 slope change was $5.9^{\circ}$ lesser in the LDD group. In the LDD group, total spinofemoral flexion was $7^{\circ}$ lesser and the lumbar spine flexion (LL change) was $16^{\circ}$ lesser, and hip flexion was $7^{\circ}$ more than that in the control group. The LDD group had an $8^{\circ}$ smaller change in the SS and a $9^{\circ}$ greater change in the PT (Fig. 2). The spine/hip ratio was significantly lower in the LDD group (0.3) than in the control group $(0.7 ; p<0.001$; Table 2$)$.

Regression analyses were performed to investigate the relationships between the spine/hip ratio and other variables. LDD group and increasing age, and not sex, height, or weight, were significant univariate predictors of a decreased spine/hip ratio (LDD group: $p<0.001$; age: $p=$ $0.048)$.

\section{Discussion}

The human body maintains the sagittal balance of the spinopelvic complex by virtue of its bony morphology and soft tissue tension. When changing position from

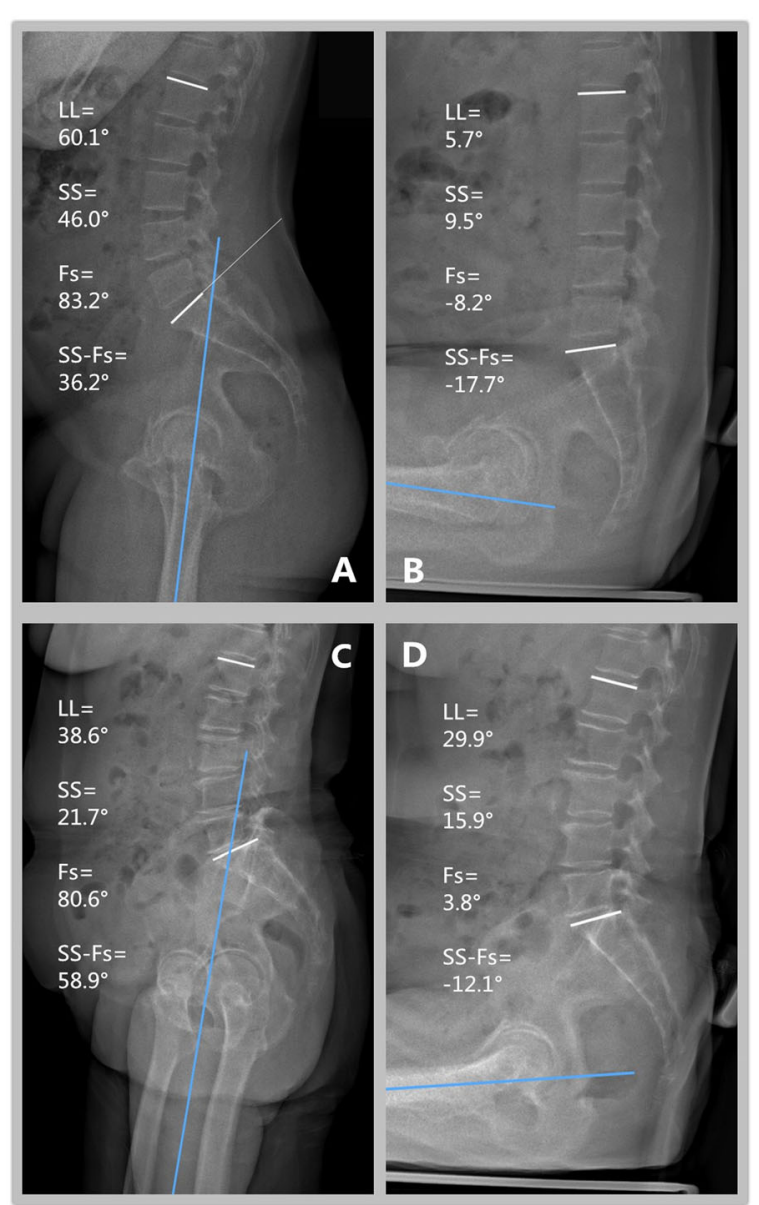

Fig. 2 Measurement of LL, SS, Fs, and SS-Fs for patient in standing and sitting position. Patients in the control group (upper A and B) had $54.4^{\circ}$ (change in $L L$ from $60.1^{\circ}$ to $5.7^{\circ}$ ) lumbar spine flexion and $53.9^{\circ}$ (change in SS-Fs from $36.2^{\circ}$ to $-17.7^{\circ}$ ) hip flexion from standing (A) to sitting (B). The pelvis rotated $36.5^{\circ}$ (change in SS from $46.0^{\circ}$ to $9.5^{\circ}$ ). Patients in the LDD group (Lower, C and D) demonstrated $8.7^{\circ}\left(38.6^{\circ}\right.$ to $\left.29.9^{\circ}\right)$ lumbar spine flexion and $71^{\circ}\left(58.9^{\circ}\right.$ to $-12.1^{\circ}$ ) hip flexion, from standing (C) to sitting (D). The pelvis rotated $5.8^{\circ}\left(21.7^{\circ}\right.$ to $\left.15.9^{\circ}\right)$

standing to sitting, the femur rotates from a vertical to a horizontal position, which is approximately $90^{\circ}$ in relation to the ground. The upper body is maintained

Table 1 Demographic data of the patients

\begin{tabular}{|c|c|c|c|c|}
\hline Parameters & Control $(n=84)$ Mean \pm SD & LDD $(n=54)$ Mean \pm SD & $p$ value & Total $(n=138)$ Mean \pm SD \\
\hline Female, number(\%) & $38(45.2 \%)$ & 35 (64.8\%) & 0.02 & $73(52.9 \%)$ \\
\hline Age (years) & $50.4 \pm 12.6$ & $63.2 \pm 9.1$ & $<0.01$ & $55.4 \pm 12.9$ \\
\hline Left hip (\%) & $38(45.2 \%)$ & $25(46.3 \%)$ & 0.90 & $63(45.7 \%)$ \\
\hline Ficat stage III, number (\%) & 79(94.0\%), & $52(96.3 \%)$ & 0.55 & $131(94.9 \%)$ \\
\hline Height (cm) & $164.1 \pm 11.9$ & $163.1 \pm 8.2$ & 0.58 & $163.7 \pm 10.6$ \\
\hline Weight (kg) & $69.3 \pm 10.7$ & $67.6 \pm 10.2$ & 0.34 & $68.7 \pm 10.5$ \\
\hline BMI $\left(\mathrm{kg} / \mathrm{m}^{2}\right)$ & $26.5 \pm 11.6$ & $25.3 \pm 3.0$ & 0.46 & $26.0 \pm 9.2$ \\
\hline
\end{tabular}


Table 2 Anatomic and positional parameters

\begin{tabular}{|c|c|c|c|c|}
\hline Parameters & Control $(n=84)$ Mean \pm SD & $\operatorname{LDD}(n=54)$ Mean \pm SD & $p$ value & Total $(n=138)$ Mean \pm SD \\
\hline PI standing $\left(^{\circ}\right)$ & $43.7 \pm 10.3$ & $46.9 \pm 11.4$ & 0.08 & $45.0 \pm 10.8$ \\
\hline PI change $\left(^{\circ}\right)$ & $-1.4 \pm 3.2$ & $-1.0 \pm 3.5$ & 0.44 & $-1.3 \pm 3.3$ \\
\hline PT standing $\left(^{\circ}\right)$ & $4.7 \pm 8.0$ & $9.2 \pm 9.0$ & 0.003 & $6.5 \pm 8.7$ \\
\hline PT change $\left(^{\circ}\right)$ & $32.5 \pm 15.3$ & $23.4 \pm 14.2$ & 0.001 & $28.9 \pm 15.7$ \\
\hline SVA standing (mm) & $22.1 \pm 31.9$ & $51.1 \pm 51.0$ & $<0.001$ & $33.5 \pm 42.7$ \\
\hline LL standing $\left(^{\circ}\right)$ & $51.0 \pm 9.7$ & $46.3 \pm 11.9$ & 0.01 & $49.2 \pm 10.8$ \\
\hline LL change (Lumbar spine flexion) $\left(^{\circ}\right)$ & $36.5 \pm 15.6$ & $20.6 \pm 13.1$ & $<0.001$ & $30.3 \pm 16.6$ \\
\hline SS standing $\left(^{\circ}\right)$ & $38.6 \pm 8.7$ & $38.1 \pm 9.0$ & 0.78 & $38.4 \pm 8.8$ \\
\hline SS change $\left(^{\circ}\right)$ & $30.5 \pm 15.5$ & $22.6 \pm 14.2$ & 0.03 & $27.4 \pm 15.4$ \\
\hline L1 slope standing $\left(^{\circ}\right)$ & $12.9 \pm 6.0$ & $10.7 \pm 6.7$ & 0.04 & $12.1 \pm 6.4$ \\
\hline L1 slope change $\left(^{\circ}\right)$ & $7.0 \pm 9.0$ & $1.1 \pm 7.4$ & $<0.001$ & $4.7 \pm 8.9$ \\
\hline Femur slope standing $\left(^{\circ}\right)$ & $87.3 \pm 4.2$ & $85.7 \pm 5.4$ & 0.06 & $86.7 \pm 4.8$ \\
\hline Femur slope change $\left({ }^{\circ}\right)$ & $86.6 \pm 5.6$ & $85.5 \pm 6.5$ & 0.30 & $86.2 \pm 6.0$ \\
\hline Hip flexion( $\left(^{\circ}\right)$ & $55.4 \pm 19.2$ & $62.3 \pm 17.4$ & 0.03 & $58.1 \pm 18.8$ \\
\hline Total spinofemoral flexion( $\left(^{\circ}\right)$ & $93.7 \pm 10.4$ & $86.5 \pm 10.3$ & $<0.001$ & $90.9 \pm 10.9$ \\
\hline Spine/Hip ratio & $0.7 \pm 0.6$ & $0.3 \pm 0.3$ & $<0.001$ & $0.6 \pm 0.5$ \\
\hline
\end{tabular}

LDD Lumbar degenerative disc disease, PI Pelvic incidence, PT Pelvic tilt, SS sacral slope, SVA Sagittal vertical axis, LL Lumbar lordosis.

upright. During this movement, the pelvis lies on both hip joints and rotates on the co-axis of the femoral heads, distributing femur movement to the trunk and over the hip joint and spine [5-8, 15, 16, 20-22]. Hip arthroplasty surgeons must identify the spinopelvic movement patterns in their patients, as patients demonstrating greater hip flexion during daily activity may be at greater risk of anterior impingement and posterior dislocation [15]. Our study assessed how the lumbar spine, pelvis, and hip joint move during change from a standing to a sitting position and the influence of lumbar disc degeneration on such spinofemoral movement.

We show that patients with LDD had $5^{\circ}$ lesser LL on standing, indicating a relatively kyphotic posture. Blizzard et al. reported that spinal deformities decreased lumbar lordosis [16]. Patients with LDD may be unable to stand erect and may walk with the trunk leaning forwards [23, 24]. Previously, Liang et al. [25] reported that patients with lumbar disc herniation, experiencing sagittal imbalance, had a significantly increased SVA of 11.6 $\mathrm{cm}$, on average. With an increased SVA and decreased LL, patients in the LDD group posteriorly rotated their pelvis by $5^{\circ}$ more (PT) in compensation.

When changing from a standing to sitting position, patients were instructed to sit on a standard chair to achieve a horizontally placed femur. Images were acquired using the EOS system to ensure reliability. The pelvis acts as a link between the upper body and lower limbs. In 1992, Duval-Beaupere et al. introduced the concept of PI as a cornerstone for describing spinofemoral relationships [26]. The PI was not significantly different between the groups in our study and did not change from the standing to the sitting position, highlighting its anatomical characteristics.

During sitting, the L1 slope change was relatively small $\left(4.7^{\circ}\right)$. As the kyphotic thoracic spine forms a cage in combination with the ribs and respiratory muscles, a minimal sagittal ROM $\left(0.1^{\circ}\right)$ is present, as reported by Ochi et al. [8] This cage provides a stable base for the cervical spine and head. Below L1, the lumbar spine, pelvis, and femur rotate in a chain-like manner to distribute the flexion of the spinofemoral movement [21]. Spinofemoral flexion is the combination of intrinsic motion of the acetabular-femur joint and extrinsic lumbar spine movements. The total lumbar spinofemoral motion for all patients in our study was $90.9^{\circ}$ on an average. For all the patients, the mean lumbar spine flexion was $30.3^{\circ}$ and hip flexion was $58.1^{\circ}$. The hip joints bear only-two thirds of spinofemoral movement from standing to sitting. The pelvis rotated $27.4^{\circ}$ usually posteriorly (change in SS) in all the patients. Many studies have demonstrated a SS change of $22^{\circ}-27^{\circ}$ from standing to sitting $[6-8,15,20]$. It has been reported that every $1^{\circ}$ increase in the pelvis posterior tilt increases the acetabular component anteversion by $0.7^{\circ}[22,27-30]$. The functional acetabular anteversion increased by $19^{\circ}\left(27.4^{\circ} \times 0.7\right)$ on average in our patients. The pelvis movement helped to prevent posterior dislocation via a combination of reduced hip movement and greater anteversion change.

Lumbar spine degeneration alters spinopelvic alignment and motion. In our study, patients in the LDD group had $7^{\circ}$ lesser total spinofemoral flexion. This was mainly due to decreased movement in the lumbar spine 
$\left(16^{\circ}\right)$. To compensate for reduced lumbar motion, patients had to recruit greater hip flexion $\left(7^{\circ}\right)$ to place the femur flat when sitting. The spine/hip ratio was significantly lower in the LDD than in the control group $(0.3$ versus $0.7 ; p<0.001)$. The LDD group had $8^{\circ}$ lesser pelvis rotation, which would decrease anteversion changes by $29.6 \%\left(8^{\circ} / 27^{\circ}\right)$.

There were more female and elderly patients in the LDD group, and the mean age of patients in this group was higher than in the control group. Multivariate analysis revealed that sex was not a significant predictor of a decreased spine/hip ratio. Moreover, the cadaver and clinical studies have not indicated a significant effect of sex on lumbar disc degeneration [31,32]. Older patients exhibit more lumbar degenerative changes, including hypertrophy of facets, degeneration of intervertebral disks, and osteophytosis of vertebrae. These phenomena would lead to disc space narrowing, loss of LL, and decreased lumbar spinal ROM. A study of 214 male patients by Burton et al. [32] revealed that reduced lumbar flexibility was multifactorial, and included lumbar disc degeneration and increased age. Furthermore, Schepper et al. reported a correlation between increased frequency of radiographic disc degeneration and age [33]. Arthroplasty surgeons should be aware that older patients with degeneration of multiple lumbar discs have a significantly different lumbar spinopelvic motion pattern.

When patients have a limited ROM in the lumbar spine, the risk of dislocation and need for revision after THA increases markedly./ Based on a 12-month follow-up, Perfetti et al. reported that, compared to the controls, the THA patients with prior spinal fusion were 7 times more likely to dislocate their prostheses $(p<0.01)$ and 4 times more likely to need revisions $(\mathrm{p}<0.01)$, [10]. Sing et al. reported that the dislocation rate was $2.4 \%$ for THA patients without prior spinal fusion, $4.3 \%$ for patients with 1 to 2 levels fused, and $7.5 \%$ for patients with 3 to 5 levels fused [11]. In this study, patients with previous spine surgery were excluded. Patients with multiple degenerative lumbar discs could be more difficult to identify than those with a clear history of spine fusion surgery. Surgeons should pay particular attention to these patients with poor spinopelvic mobility, as they have greater hip flexion, increasing their risk of impingement and posterior dislocation. As both patients and surgeons are increasingly more prone to relax hip precautions postoperatively, our study outcomes may help surgeons to identify the THA candidates with stiff lumbar spine movements preoperatively. However, methods to identify these patients more easily than by Xray imaging while standing and sitting need further investigation. Surgeons may have to place the prosthesis in a more individualized position during surgery; this has gained increased attention. It is necessary to preparing special implants, such as a dural-mobility cup, in advance, particularly when patients have additional risk factors for dislocation. Personized postoperative rehabilitation protocols should be prescribed in these cases.

This study had several limitations. First, static standing and sitting images do not fully represent a patient's pelvic orientation during activities of daily living. Patients usually had their hip dislocated posteriorly in a specific position involving flexion, adduction, and internal rotation. Further research is required to evaluate this issue. Dislocation on sitting was rare, unless the hip was highly unstable. We did not perform assessments in a squatting position as painful hip joints hindered patients from squatting fully prior to surgery. We have been followingup these patients to record any postoperative dislocation and to relate our measurements to clinical outcomes. Second, patients preparing for THA may have stiff and painful hip motion, leading to increased lumbar spine flexion on sitting. There was no difference in the AVN stage between the two groups, and the majority of the patients were of FICAT Stage III AVN; this helped mitigate this bias. Third, the quantitative influence of the severity of lumbar disc degeneration on spinofemoral movement was not included. More patients and longer follow-up are required to analyze these differences.

\section{Conclusions}

In conclusion, on standing, patients with LDD exhibited greater forward trunk leaning than those without LDD and had a larger compensatory pelvis posterior tilt angle. On sitting, the LDD group had reduced lumbar spine/hip flexion ratio, demonstrating greater hip joint flexion in compensation for reduced lumbar spine flexion. Surgeons should take note that older patients with LDD affecting multiple discs are at greater risk of anterior impingement and posterior dislocation after THA. Preoperative identification of such patients, intraoperative surgical technique modification, and individualized rehabilitation protocols are necessary for improving outcomes.

\begin{abstract}
Abbreviations
AP: Anteroposterior; AVN: Avascular necrosis; AVNFH: Avascular necrosis of the femoral head; Fs: Femoral slope; L1 s: L1 slope; LDD: Lumbar degenerative disc disease; LL: Lumbar lordosis angle; PI: Pelvic incidence; PT: Pelvic tilt; ROM: Range of motion; SS: Sacral slope; SVA: Sagittal vertical axis; THA: Total hip arthroplasty; UCLA: University of California at Los Angeles
\end{abstract}

\section{Acknowledgements}

Not applicable.

Authors' contributions

JG and YZ conceptualized the study; JG, HF, and XF collected and analyzed the data; JG, HF, XF, and $Y Z$ interpreted the results; JG and HF wrote the paper; all authors discussed the results and revised the manuscript.

Funding

This study was supported financially by Beijing Jishuitan Hospital (XKGG201810 to JG). The funding body had no role in the study design, data collection, analyses, or interpretation, or in writing the manuscript. 


\section{Availability of data and materials}

The datasets used and/or analyzed during the current study are available from the corresponding author on reasonable request.

\section{Ethics approval and consent to participate}

This retrospective study was approved by the Beijing Jishuitan Hospital Institutional Review Board (Beijing Jishuitan Hospital Institutional Review Board Approval Form No.201812-09) and was carried out in accordance with the ethical principles of the Declaration of Helsinki. Data were retrieved by medical records review and all activities associated with this research project were performed in accordance with Jishuitan Hospital Institutional Guidelines and Clinical Regulations. Verbal informed consent was obtained from participants or their guardians.

\section{Consent for publication}

Not applicable.

\section{Competing interests}

The authors declare that they have no competing interests.

Received: 27 June 2019 Accepted: 6 January 2020

Published online: 18 January 2020

\section{References}

1. Kummer FJ, Shah S, lyer S, DiCesare PE. The effect of acetabular cup orientations on limiting hip rotation. J Arthroplast. 1999;14(4):509-13.

2. Lewinnek GE, Lewis JL, Tarr R, Compere CL, Zimmerman JR. Dislocations after total hip-replacement arthroplasties. J Bone Joint Surg Am. 1978;60(2): 217-20.

3. Widmer $\mathrm{KH}$, Zurfluh B. Compliant positioning of total hip components for optimal range of motion. J Orthop Res. 2004;22(4):815-21.

4. Abdel MP, von Roth P, Jennings MT, Hanssen AD, Pagnano MW. What safe zone? The vast majority of dislocated THAs are within the Lewinnek safe zone for Acetabular component position. Clin Orthop Relat Res. 2016;474(2): 386-91.

5. Babisch JW, Layher F, Amiot LP. The rationale for tilt-adjusted acetabular cup navigation. J Bone Joint Surg Am. 2008:90(2):357-65.

6. Kanawade V, Dorr LD, Wan Z. Predictability of Acetabular component angular change with postural shift from standing to sitting position. J Bone Joint Surg Am. 2014;96(12):978-86.

7. Loppini M, Longo UG, Ragucci P, Trenti N, Balzarini L, Grappiolo G. Analysis of the pelvic functional orientation in the sagittal plane: a radiographic study with EOS 2D/3D technology. J Arthroplast. 2017;32(3):1027-32.

8. Ochi H, Baba T, Homma Y, Matsumoto M, Nojiri H, Kaneko K. Importance of the spinopelvic factors on the pelvic inclination from standing to sitting before total hip arthroplasty. Eur Spine J : Official Publication Eur Spine Soc, Eur Spinal Deformity Soc, Eur Sect Cervical Spine Res Soc. 2016;25(11):3699706.

9. McGregor AH, McCarthy ID, Dore CJ, Hughes SP. Quantitative assessment of the motion of the lumbar spine in the low back pain population and the effect of different spinal pathologies of this motion. Eur Spine J. 1997;6(5): 308-15.

10. Perfetti DC, Schwarzkopf R, Buckland AJ, Paulino CB, Vigdorchik JM. Prosthetic Dislocation and Revision After Primary Total Hip Arthroplasty in Lumbar Fusion Patients: A Propensity Score Matched-Pair Analysis. J Arthroplasty. 2017;32(5):1635-1640.e1631.

11. Sing DC, Barry JJ, Aguilar TU, Theologis AA, Patterson JT, Tay BK, Vail TP, Hansen EN. Prior Lumbar Spinal Arthrodesis Increases Risk of ProstheticRelated Complication in Total Hip Arthroplasty. J Arthroplasty. 2016;31(9 Suppl):227-232.e221.

12. Ficat RP. Idiopathic bone necrosis of the femoral head. Early diagnosis and treatment. J Bone Joint Surg Br. 1985;67(1):3-9.

13. Deschenes S, Charron G, Beaudoin G, Labelle H, Dubois J, Miron MC, Parent S. Diagnostic imaging of spinal deformities: reducing patients radiation dose with a new slot-scanning X-ray imager. Spine (Phila Pa 1976). 2010; 35(9):989-94.

14. Wade R, Yang H, McKenna C, Faria R, Gummerson N, Woolacott N. A systematic review of the clinical effectiveness of EOS 2D/3D X-ray imaging system. Eur Spine J. 2013;22(2):296-304.

15. Esposito Cl, Miller TT, Kim HJ, Barlow BT, Wright TM, Padgett DE, Jerabek SA Mayman DJ. Does degenerative lumbar spine disease influence
Femoroacetabular flexion in patients undergoing Total hip Arthroplasty? Clin Orthop Relat Res. 2016:474(8):1788-97.

16. Blizzard DJ, Nickel BT, Seyler TM, Bolognesi MP. The impact of lumbar spine disease and deformity on Total hip Arthroplasty outcomes. Orthop Clin North Am. 2016;47(1):19-28.

17. Akbar M, Terran J, Ames CP, Lafage V, Schwab F. Use of Surgimap spine in sagittal plane analysis, osteotomy planning, and correction calculation. Neurosurg Clin N Am. 2013;24(2):163-72.

18. Cheh G, Bridwell KH, Lenke LG, Buchowski JM, Daubs MD, Kim Y, Baldus C. Adjacent segment disease followinglumbar/thoracolumbar fusion with pedicle screw instrumentation: a minimum 5-year follow-up. Spine (Phila Pa 1976). 2007;32(20):2253-7.

19. Ghiselli G, Wang JC, Bhatia NN, Hsu WK, Dawson EG. Adjacent segment degeneration in the lumbar spine. J Bone Joint Surg Am. 2004;86-a(7):1497503.

20. Legaye J. Influence of the sagittal balance of the spine on the anterior pelvic plane and on the acetabular orientation. Int Orthop. 2009;33(6):1695700.

21. Lum ZC, Coury JG, Cohen JL, Dorr LD. The current knowledge on Spinopelvic mobility. J Arthroplast. 2018;33(1):291-6.

22. Maratt JD, Esposito Cl, McLawhorn AS, Jerabek SA, Padgett DE, Mayman DJ. Pelvic tilt in patients undergoing total hip arthroplasty: when does it matter? J Arthroplast. 2015;30(3):387-91.

23. Jackson RP, McManus AC. Radiographic analysis of sagittal plane alignment and balance in standing volunteers and patients with low back pain matched for age, sex, and size. Prospective Control Clin Study Spine (Phila Pa 1976). 1994;19(14):1611-8.

24. Schwab FJ, Blondel B, Bess S, Hostin R, Shaffrey Cl, Smith JS, Boachie-Adjei O, Burton DC, Akbarnia BA, Mundis GM, et al. Radiographical spinopelvic parameters and disability in the setting of adult spinal deformity: a prospective multicenter analysis. Spine (Phila Pa 1976). 2013;38(13):E803-12.

25. Liang C, Sun J, Cui X, Jiang Z, Zhang W, Li T. Spinal sagittal imbalance in patients with lumbar disc herniation: its spinopelvic characteristics, strength changes of the spinal musculature and natural history after lumbar discectomy. BMC Musculoskelet Disord. 2016;17:305.

26. Duval-Beaupere G, Schmidt C, Cosson P. A Barycentremetric study of the sagittal shape of spine and pelvis: the conditions required for an economic standing position. Ann Biomed Eng. 1992;20(4):451-62.

27. Lembeck B, Mueller O, Reize P, Wuelker N. Pelvic tilt makes acetabular cup navigation inaccurate. Acta Orthop. 2005;76(4):517-23.

28. Ranawat CS, Ranawat AS, Lipman JD, White PB, Meftah M. Effect of spinal deformity on pelvic orientation from standing to sitting position. J Arthroplast. 2016;31(6):1222-7.

29. Wan Z, Malik A, Jaramaz B, Chao L, Dorr LD. Imaging and navigation measurement of acetabular component position in THA. Clin Orthop Relat Res. 2009;467(1):32-42

30. Zhu J, Wan Z, Dorr LD. Quantification of pelvic tilt in total hip arthroplasty. Clin Orthop Relat Res. 2010;468(2):571-5.

31. Siemionow K, An H, Masuda K, Andersson G, Cs-Szabo G. The effects of age, sex, ethnicity, and spinal level on the rate of intervertebral disc degeneration: a review of 1712 intervertebral discs. Spine. 2011;36(17):13339.

32. Burton AK, Battie MC, Gibbons L, Videman T, Tillotson KM. Lumbar disc degeneration and sagittal flexibility. J Spinal Disord. 1996;9(5):418-24.

33. de Schepper El, Damen J, van Meurs JB, Ginai AZ, Popham M, Hofman A, Koes BW, Bierma-Zeinstra SM. The association between lumbar disc degeneration and low back pain: the influence of age, gender, and individual radiographic features. Spine. 2010;35(5):531-6.

\section{Publisher's Note}

Springer Nature remains neutral with regard to jurisdictional claims in published maps and institutional affiliations. 